\title{
Fluorescence grid analysis for the evaluation of piecemeal surgery in sinonasal inverted papilloma: a proof-of-concept study
}

\author{
J Vonk $^{1} \cdot$ FJ Voskuil $^{1,2} \cdot$ JG de Wit $^{1} \cdot$ WT Heeman $^{3,4,5} \cdot$ WB Nagengast $^{6} \cdot$ GM van Dam $^{3,7} \cdot$ RA Feijen $^{8}$. \\ AGW Korsten-Meijer ${ }^{8} \cdot$ B van der Vegt $^{2} \cdot$ MJH Witjes $^{1}$
}

Received: 27 May 2021 / Accepted: 17 September 2021 / Published online: 5 November 2021

(c) The Author(s) 2021

\begin{abstract}
Purpose Local recurrence occurs in 19\% of sinonasal inverted papilloma (SNIP) surgeries and is strongly associated with incomplete resection. During surgery, it is technically challenging to visualize and resect all SNIP tissue in this anatomically complex area. Proteins that are overexpressed in SNIP, such as vascular endothelial growth factor (VEGF), may serve as a target for fluorescence molecular imaging to guide surgical removal of SNIP. A proof-of-concept study was performed to investigate if the VEGF-targeted near-infrared fluorescent tracer bevacizumab-800CW specifically localizes in SNIP and whether it could be used as a clinical tool to guide SNIP surgery.

Methods In five patients diagnosed with SNIP, $10 \mathrm{mg}$ of bevacizumab-800CW was intravenously administered 3 days prior to surgery. Fluorescence molecular imaging was performed in vivo during surgery and ex vivo during the processing of the surgical specimen. Fluorescence signals were correlated with final histopathology and VEGF-A immunohistochemistry. We introduced a fluorescence grid analysis to assess the fluorescence signal in individual tissue fragments, due to the nature of the surgical procedure (i.e., piecemeal resection) allowing the detection of small SNIP residues and location of the tracer ex vivo. Results In all patients, fluorescence signal was detected in vivo during endoscopic SNIP surgery. Using ex vivo fluorescence grid analysis, we were able to correlate bevacizumab-800CW fluorescence of individual tissue fragments with final histopathology. Fluorescence grid analysis showed substantial variability in mean fluorescence intensity $\left(F I_{\text {mean }}\right)$, with SNIP tissue showing a median $F I_{\text {mean }}$ of 77.54 (IQR 50.47-112.30) compared to 35.99 (IQR 21.48-57.81) in uninvolved tissue $(p<0.0001)$, although the diagnostic ability was limited with an area under the curve of 0.78 .

Conclusions A fluorescence grid analysis could serve as a valid method to evaluate fluorescence molecular imaging in piecemeal surgeries. As such, although substantial differences were observed in fluorescence intensities, VEGF-A may not be the ideal target for SNIP surgery.
\end{abstract}

Trial registration NCT03925285.

Keywords Paranasal sinus neoplasms $\cdot$ Papilloma $\cdot$ Inverted $\cdot$ Optical imaging $\cdot$ Molecular imaging $\cdot$ Vascular endothelial growth factor A

This article is part of the Topical Collection on Oncology-Head and Neck

van der Vegt B. and Witjes M.J.H. contributed equally to this work and share last authorship of this article

MJH Witjes

m.j.h.witjes@umcg.nl

Extended author information available on the last page of the article

\section{Introduction}

Sinonasal inverted papilloma (SNIP) is a benign tumor; yet, it is characterized by an aggressive growth pattern with destruction of adjacent bone, co-existing chronic inflammation, and risk of malignant transformation to squamous cell carcinoma [1,2]. Even though the clinical features of SNIP are well described, the exact etiology of SNIP remains unclear [3]. The main treatment strategy of SNIP consists of endoscopic surgical resection to remove all SNIP tissue, and in more progressed stages even resecting the underlying bone at the insertion point to remove microscopic mucosal 
residues $[1,4]$. Despite performing a thorough resection, local recurrence rates of up to $19 \%$ have been reported $[5,6]$, of which incomplete resection is considered to be the main causal factor [7]. This illustrates the unmet clinical need for tools that can improve visualization of all SNIP tissue in this anatomically complex area and aid in achieving a complete resection.

Fluorescence molecular imaging (FMI) is an emerging surgical guidance technique that has gained increasing interest in head and neck surgery [8]. FMI provides highresolution visualization of disease-specific biomarkers and has been shown to adequately discriminate target tissue from surrounding tissues for various indications [9-13]. Therefore, it is of interest to evaluate the potential of FMI to highlight in vivo SNIP tissue intraoperatively and provide real-time feedback on excised tissue during SNIP surgery. The main biomarkers that have been associated with SNIP pathogenesis and may serve as a target for FMI include cyclooxygenase [14], epidermal growth factor [15, 16], and vascular endothelial growth factor (VEGF) [17-19]. It was shown that VEGF, a key mediator in angiogenesis related to inflammation and uncontrolled cell growth, correlated the Krouse classification system for assessment of resectability [19]. Consequently, targeted imaging of these biomarkers of SNIP might assist the surgeon in more complete resection. FMI using the near-infrared fluorescent tracer bevacizumab-800CW targeting the soluble ligand VEGF-A has been performed successfully for perioperative fluorescence detection and delineation in various solid tumors, including both malignant and benign tumors [20-23].

This study aimed to determine if the intravenously administered NIR fluorescent tracer bevacizumab-800CW accumulates in SNIP and can be used to discriminate between SNIP and uninvolved tissue, which may aid in radical resection of SNIP. Therefore, we studied the feasibility for clinical use of bevacizumab-800 CW fluorescence both in vivo during endoscopic SNIP surgery and ex vivo by imaging the freshly excised tissue fragments after piecemeal surgery.

\section{Materials and methods}

\section{Study design and patients}

This proof-of-concept study was performed by the Departments of Otolaryngology and Oral \& Maxillofacial Surgery of the University Medical Center Groningen (UMCG). Patients $>18$ years old scheduled for surgical removal of histologically confirmed SNIP were enrolled in the study. The study was performed in concordance with the Declaration of Helsinki (adapted version Fortaleza, Brazil, 2013) and the Dutch Act on Medical Research involving Human Subjects. Approval was obtained at the Institutional
Review Board of the UMCG (NL66494.042.18). Informed consent was obtained from all subjects prior to any study procedure. The trial was registered at www.clinicaltrials.gov (NCT03925285).

\section{The fluorescent tracer bevacizumab-800 CW}

Clinical-grade bevacizumab-800CW was manufactured in the good manufacturing practice unit of the UMCG, as previously described $[24,25]$. Briefly, bevacizumab (Roche, AG) and IRDye-800CW-NHS (LI-COR Biosciences, NE, USA) were conjugated to a dye to antibody ratio of 2:1. Subsequently, bevacizumab- $800 \mathrm{CW}$ was formulated in a sodium chloride solution at a concentration of $1 \mathrm{mg} / \mathrm{mL}$. Based on prior clinical trial findings with the tracer [20, 22, 26], a dose of $10 \mathrm{mg}$ was chosen for the current study, based on an optimal target-to-background ratio (TBR) obtained in vivo and limited variance in tumor $F I_{\text {mean }}$.

Three days prior to surgery, patients were administered a bolus injection of $10 \mathrm{mg}$ bevacizumab-800CW intravenously. Patients were monitored after tracer administration for $1 \mathrm{~h}$, and adverse events were reported according to the Common Terminology Criteria for Adverse Events (CTCAE) version 4.0. The study workflow is summarized in Fig. 1.

\section{Intraoperative imaging}

All subjects underwent endoscopic surgery according to standard of care. Before resection, FMI of all locations (e.g., maxillary sinus, nasal cavity) comprising SNIP tissue was performed, using the contralateral (i.e., unaffected) side as a negative control. After resection, nasal pledgets soaked in cocaine/adrenaline were introduced to stop bleeding and temporarily removed to obtain fluorescence images of the wound beds. Immediately after resection, all tissue fragments including both SNIP and uninvolved tissue (i.e., normal mucosa, reactive mucosa, and connective tissue) were imaged during surgery at the back-table using a closed-field fluorescence imaging system (Pearl Trilogy, LI-COR Biosciences, NE, USA).

\section{Ex vivo validation}

After surgery and closed-field imaging in the Pearl imaging system, all tissue fragments were submitted to the Department of Pathology, formalin-fixed and paraffin-embedded (FFPE) in tissue blocks. FFPE blocks were imaged with the Odyssey CLX ${ }^{\circledR}$ flatbed scanner (LI-COR Biosciences Inc., NE, USA) to further perform in-depth analysis of the localization of the fluorescence signals. Subsequently, $3-\mu \mathrm{m}$ tissue sections were cut for hematoxylin and eosin (H\&E) staining and were digitalized for further analysis. 


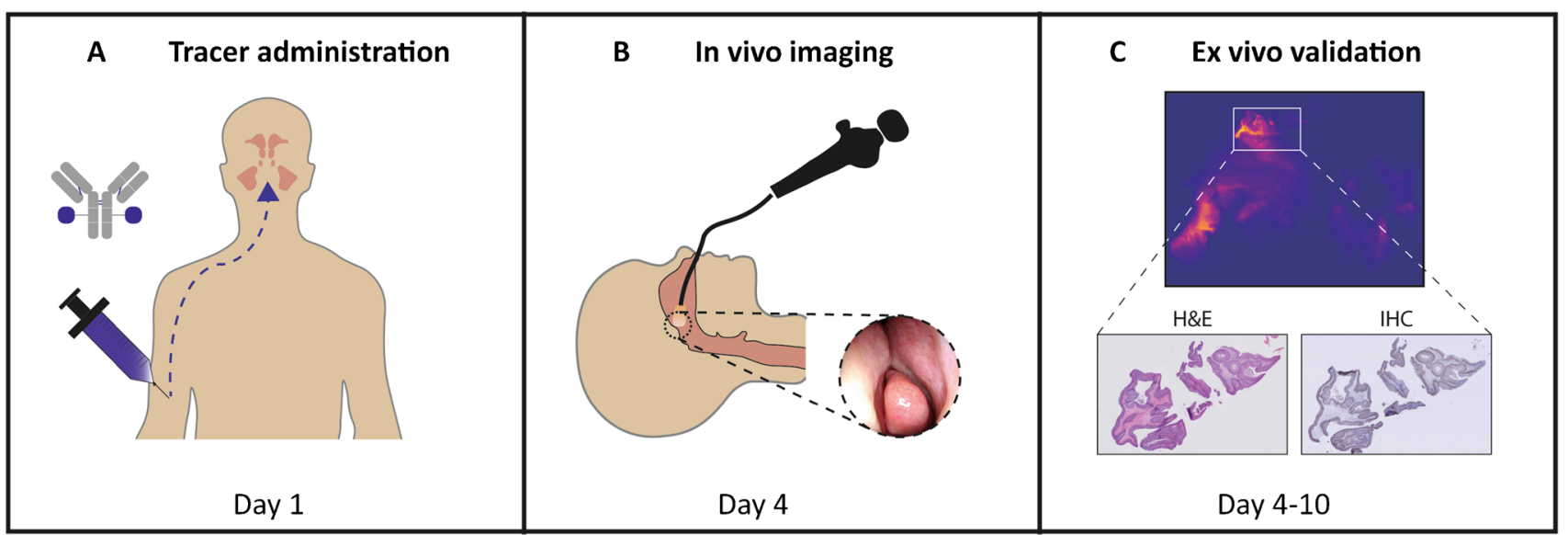

Fig. 1 Summary of study workflow. A Bevacizumab-800CW is administered intravenously through a bolus injection. B Three days after administration, in vivo fluorescence molecular imaging is performed during surgery to study the clinical use. C During tissue processing, ex vivo fluorescence molecular imaging of all FFPE blocks

Our standard analysis of FMI consisted of drawing regions of interest (ROI) containing SNIP on the 3- $\mu \mathrm{m}$ H\&E sections by a head and neck pathologist blinded for FMI results. ROIs were superimposed on the fluorescence is performed. Ultimately, fluorescence signal is correlated with final H\&E histopathology (i.e., presence of SNIP) and VEGF-A immunohistochemistry. Abbreviations: FFPE, formalin-fixed, paraffin-embedded; H\&E, hematoxylin and eosin; SNIP, sinonasal inverted papilloma; VEGF-A, vascular endothelial growth factor A

flatbed scan of the corresponding FFPE block, and the $F I_{\text {mean }}$ of SNIP and uninvolved tissue were determined (Fig. 2A). A TBR was calculated by dividing the target ROI $\left(F I_{\text {mean }}\right.$ SNIP) with the background ROI ( $F I_{\text {mean }}$ uninvolved tissue)
Fig. 2 Ex vivo fluorescence molecular imaging analyses. A Using the standard fluorescence analysis, H\&E segmentation of SNIP and uninvolved tissue are superimposed on the fluorescence image of the corresponding FFPE block. This method renders one value of both SNIP (blue ROI) and uninvolved tissue (orange ROI) per FFPE block, in which variability in fluorescence intensity is undesirably averaged. B In the fluorescence grid analysis, a $25 \times 25$ pixel grid is rendered on the fluorescence image. Using H\&E segmentation as a reference, each square is scored as SNIP or uninvolved tissue, excluding squares that do not completely comprise tissue. Mean fluorescence intensity is calculated per square, resulting in multiple data points that demonstrate variability in signal. Abbreviations: FFPE, formalin-fixed, paraffin-embedded; SNIP, sinonasal inverted papilloma; TBR, target-tobackground ratio

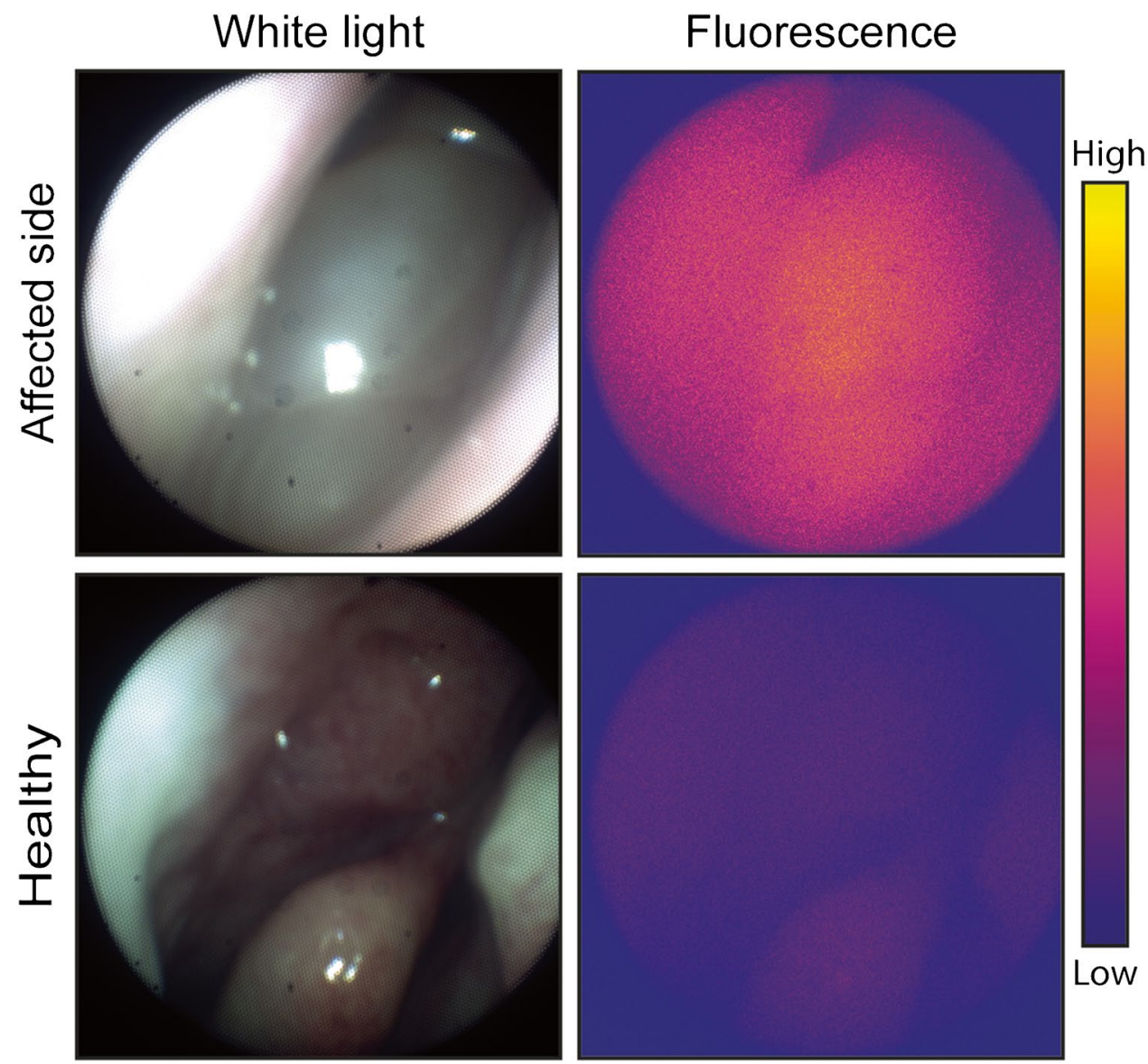


per tissue slice. Median TBR values were presented per patient. Additional VEGF-A immunohistochemistry was performed per patient on two tissue sections that each contained both SNIP and uninvolved tissue. Fluorescence flatbed scans were used to correlate bevacizumab-800CW fluorescence and immunohistochemical VEGF-A expression qualitatively.

\section{Fluorescence grid analysis}

Due to the nature of the surgical SNIP procedure, i.e., piecemeal resection, a requirement for the clinical use of FMI is identifying the nature of the small tissue fragments. Consequently, some of the tissue fragments contain normal tissue as well as SNIP or separately and not en bloc as seen in complete surgical resections. To better study the fluorescence signal within all tissue fragments, a grid of $25 \times 25$ pixels was used as an overlay for fluorescence images that have been obtained with the Odyssey CLX® flatbed scanner [30]. Each square was scored as either SNIP or uninvolved tissue based on the assessment of the pathologist (BvdV). Only the squares that completely comprised SNIP or uninvolved tissue were included in the analysis. The mean fluorescence intensity $\left(F I_{\text {mean }}\right)$ was automatically calculated with MATLAB (versionR2020a, The MathWorks, Natick, USA) for each square (Fig. 2B).

\section{Statistical analysis}

Descriptive statistics were performed on patient demographics. Given the small sample size, individual data were presented in tabular formats. Due to the limited sample size, all data was considered non-normally distributed. Imaging data were presented as median with range or interquartile range (IQR). $F I_{\text {mean }}$ was defined as total counts per ROI pixel area (signal/pixel), and calculated in ImageJ (Fiji, version 2.0.0) for standard fluorescence analysis. Fluorescence grid data was generated using MATLAB (versionR2020a, The MathWorks,
Natick, USA), and the $F I_{\text {mean }}$ per square was calculated automatically. For comparison of fluorescence imaging data, the Mann-Whitney $U$ test was used. Cutoff values of fluorescence grid analysis were determined based on Youden's $J$ statistics. Sensitivity, specificity, and accuracy were calculated using standard formulas. A two-sided $p$-value $<0.05$ was considered statistically significant. Statistical analyses and graph designs were performed using GraphPad Prism (version 9.0, GraphPad Software Inc., San Diego, CA, USA).

\section{Results}

Between January 2019 and November 2020, six subjects were included in the study. One subject did not show SNIP on final histopathology and was used as a reference. No malignant transformation of SNIP was observed. Patient demographics and clinical features are summarized in Table 1. All patients received a single bolus injection of $10 \mathrm{mg}$ bevacizumab-800CW without any side effects.

\section{In vivo fluorescence-guided endoscopic surgery}

All five subjects underwent endoscopic endonasal surgery without conversion to an open procedure. In all cases, in vivo imaging was performed, and if applicable, multiple locations containing SNIP were inspected before resection. Endoscopic detection of fluorescence signal was possible in all patients; however, TBRs could not be calculated since SNIP could not be reliably differentiated from uninvolved tissue, which is a necessity for calculating tumor to normal ratios in vivo. Qualitatively, the affected side showed higher fluorescence than the healthy side for all five patients (Fig. 3). Imaging of the wound bed did not result in useable images for analysis due to the presence of blood.

Table 1 Patient and clinical characteristics of study population

\begin{tabular}{|c|c|c|c|c|c|c|}
\hline & 1 & 2 & 3 & 4 & 5 & 6 \\
\hline Gender & Male & Female & Male & Male & Male & Male \\
\hline Age & 68 & 49 & 54 & 72 & 49 & 57 \\
\hline Insertion site & Nasal cavity & Unknown* & Ethmoid & Maxillary & Ethmoid & Nasal cavity \\
\hline Number of prior surgeries & 1 & 1 & 1 & 1 & 2 & 2 \\
\hline Surgical approach & Endoscopic & Endoscopic & Endoscopic & Endoscopic & Endoscopic & Endoscopic \\
\hline Final pathology & SNIP & $\begin{array}{l}\text { SNIP } \\
\text { No }\end{array}$ & $\begin{array}{l}\text { SNIP } \\
\text { No }\end{array}$ & SNIP & Chronic inflammation & SNIP \\
\hline Malignant transformation & No & No & No & No & No & No \\
\hline
\end{tabular}

*The insertion site could not be determined during surgery. Abbreviations: SNIP, sinonasal inverted papilloma 


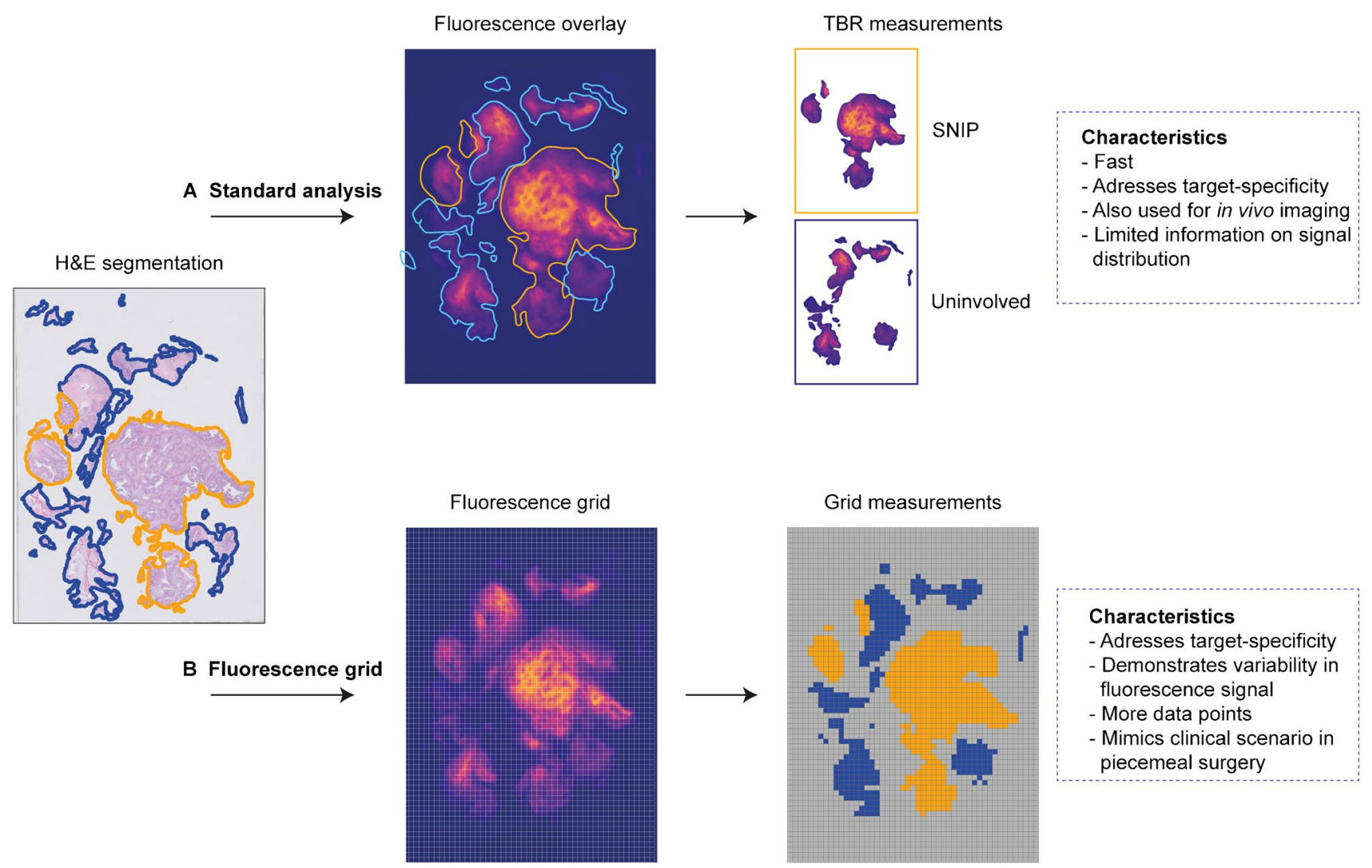

Fig. 3 In vivo visualization of fluorescence during endoscopic sinus surgery. Representative images obtained with the nasoendoscopic fluorescence imaging system. The affected side shows higher fluorescence signal compared to the contralateral healthy side. Both inverted

\section{Ex vivo fluorescence molecular imaging of bevacizumab-800CW}

To determine the potential of bevacizumab-800CW for the discrimination between SNIP and uninvolved tissue, we calculated the $F I_{\text {mean }}$ of the FFPE tissue blocks $(n=61)$. As most tissue sections contained both SNIP and uninvolved tissue, a total of 30 FFPE blocks containing SNIP and 52 containing uninvolved tissue were identified. Median $F I_{\text {mean }}$ in SNIP was 86.88 (IQR 67.57-101.20) compared to 38.30 (IQR 22.28-57.99) in uninvolved tissue $(p<0.0001)$, although substantial variation was observed within and between patients. The median $F I_{\text {mean }}$ and corresponding TBRs per patient are shown in Fig. 4A.

We studied the variability of fluorescence signal between different tissue fragments in more detail using the $25 \times 25$ pixel grid analysis. A total of 202,752 grid squares was rendered, of which 30,425 completely comprised tissue, with 13,454 classified as SNIP and 28,025 as uninvolved tissue based on H\&E histopathology. As such, 2973-22,062 measurements per patient were obtained to study the fluorescence signal (Fig. 2B). Again, higher median $F I_{\text {mean }}$ was observed papilloma and chronic inflammation were observed at final histopathology, so it is unclear whether fluorescence signal at the affected side is specific for inverted papilloma

in SNIP; 77.54 (IQR 50.47-112.30) compared to uninvolved tissue 35.99 (IQR 21.48-57.81) $(p<0.0001)$. Even though similar TBRs were obtained, the fluorescence grid analysis showed greater variability in fluorescence intensity (Fig. 4B). The ROC curve for all patients combined showed an area under the curve of 0.78 (Supplemental Fig. 1). The optimal cutoff value of $52.80 F I_{\text {mean }}$ rendered $72.71 \%$ sensitivity and $70.72 \%$ specificity, with a positive predictive value and negative predictive value of $54.39 \%$ and $84.37 \%$, respectively.

\section{Distribution of bevacizumab-800CW in SNIP at a microscopic level}

VEGF immunohistochemistry of tissue sections was performed and compared to fluorescence flatbed scans of the corresponding FFPE-block to study co-localization of bevacizumab-800CW with VEGF-A expression. Four of five patients showed VEGF-A expression. Increased fluorescence signal was observed in, but not limited to, regions showing VEGF-A expression. The regions that showed increased VEGF-A expression were not only limited to SNIP but also included reactive stroma and submucosa with an abundance of plasma cells in VEGF-A positive stroma. In 


\section{A Standard fluorescence analysis}

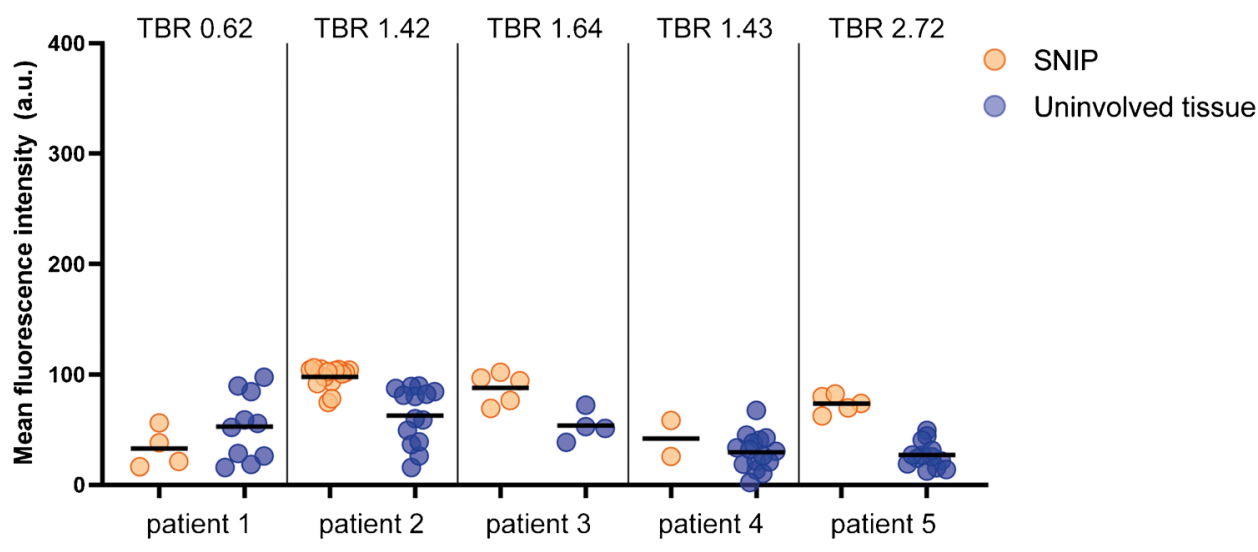

B Fluorescence grid analysis

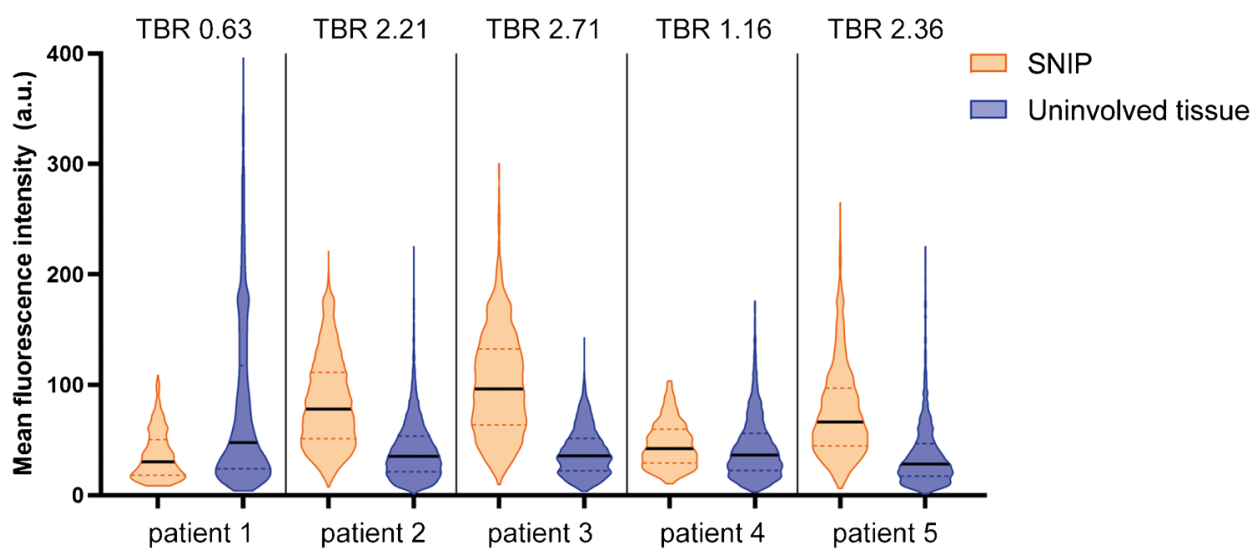

Fig. 4 Fluorescence molecular imaging of formalin-fixed, paraffinembedded tissue. A Scatter plot of the standard fluorescence analysis. Each circle represents the $F I_{\text {mean }}$ of a single FFPE block $(n=61)$, with some FFPE blocks comprising both SNIP and uninvolved tissue. For each patient, the TBR is shown above the corresponding dots. Patient 1 did not show VEGF-A expression. B Violin plot of the $F I_{\text {mean }}$ observed with fluorescence grid analysis. Because of the large amount of data points, data is visualized using violin plots instead of scatter plots. The $F I_{\text {mean }}$ of all squares $(n=30,425)$ comprising either SNIP or uninvolved mucosa are shown. Albeit the TBRs of the fluorescence grid analysis are different from standard fluorescence analy-

addition, regions showing edema and increase in microvasculature showed increased fluorescence signal despite absent or low VEGF-A expression. A representative image of VEGF-A immunohistochemistry and co-localization with fluorescence flatbed scanning is shown in Fig. 5.

\section{Discussion}

To our knowledge, this is the first clinical study that investigates the potential of FMI in SNIP using the VEGF-targeted antibody bevacizumab-800CW. Here, we show an sis, the main difference between the two analysis methods is that the fluorescence grid analysis better shows the variability in fluorescence intensity. As such, whereas both methods show a notable difference in fluorescence intensity between SNIP and uninvolved mucosa, the fluorescence grid analysis better evaluates the imaging approach in the light of piecemeal surgery, which requires assessment of individual tissue fragments. Abbreviations: $F I_{\text {mean }}$, mean fluorescence intensity; FFPE, formalin-fixed, paraffin-embedded; TBR, target-to-background ratio; VEGF-A, vascular endothelial growth factor A; SNIP, sinonasal inverted papilloma

increased fluorescence signal in SNIP compared to uninvolved tissue, when using both the standard fluorescence analysis and the fluorescence grid analysis. In addition, all patients displayed visually more in vivo fluorescence signal of the affected side compared to the contralateral healthy side. However, it was not possible to attribute the observed higher fluorescence signal during endoscopic imaging to SNIP because SNIP could not be differentiated from adjacent inflammatory mucosa based on clinical assessment.

Throughout the current study, we have used several outcome parameters to study FMI using bevacizumab-800CW 

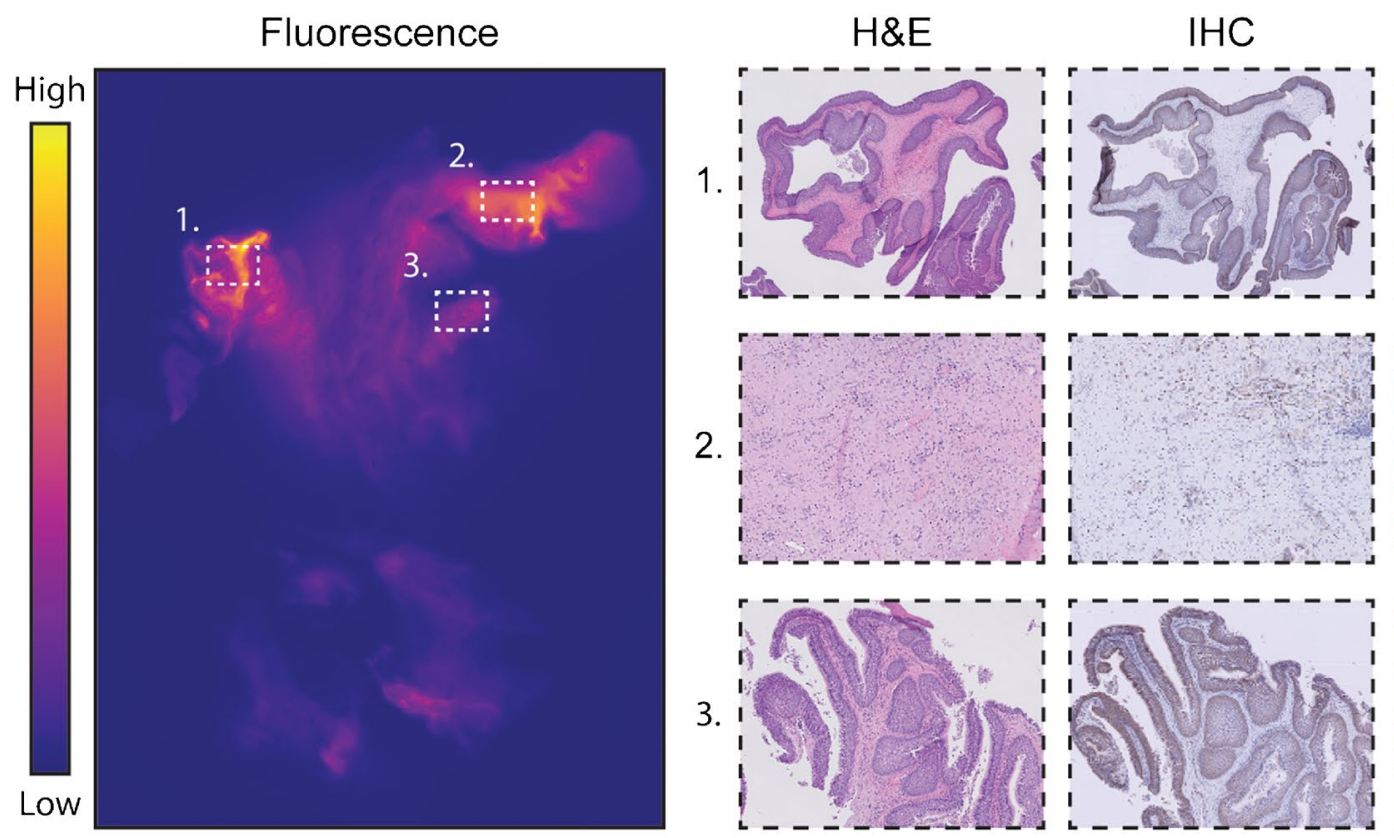

Overlay
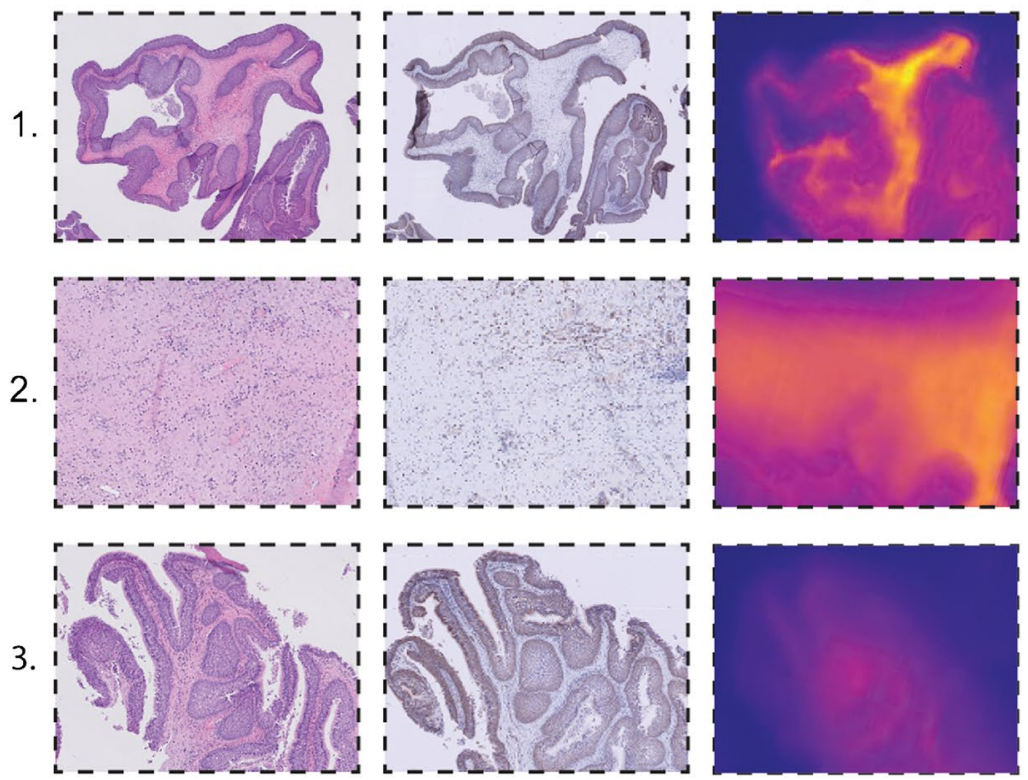

Fig. 5 Ex vivo validation. To study the microscopic tracer distribution of bevacizumab-800CW, fluorescence images were correlated with corresponding VEGF-A IHC and H\&E tissue sections. We observed an increased fluorescence signal in regions showing VEGFA expression. These regions were not only limited to SNIP (1) but also included reactive stroma and submucosa with an abundance of plasma cells that expressed VEGF-A (2). Variability in fluorescence signal was observed between different regions of SNIP $(1,3)$. In addition, regions including edema and increased vasculature also showed increased fluorescence signal despite absence of VEGF expression (not shown in figure). Abbreviations: VEGF-A, vascular endothelial growth factor $\mathrm{A}$; IHC, immunohistochemistry; H\&E, hematoxylin and eosin

suggesting that the fluorescence grid analysis can help in evaluating FMI for clinical use, thereby providing substantiated go/no-go decisions in phase I/II studies. We ascertained that the standard method of evaluating FMI for wide local excisions is not preferable for piecemeal surgery since it masks the (substantial) variability present in the imaging data as only two ROIs (i.e., target and background) are obtained per FFPE block. Therefore, we have developed a fluorescence grid analysis that may serve as a structured method for evaluating novel compounds in early-phase FMI studies in piecemeal surgery without interfering with the current analytical frameworks used for FMI.

Although multiple clinical studies have shown successful use of FMI in the removal of solid tumors, piecemeal surgery may require a different approach. The microscopically heterogeneous expression of the target that is typically observed does not per se impede guiding the surgeon in resection of bulk tissue (i.e., wide local excision) due to the margin of primary interest. In contrast, in piecemeal surgery, removal of microscopic residues is more critical and macroscopic imaging of a heterogeneous pattern may be insufficient. Here, microscopic small tissue fragments must be independently assessed for the presence of the disease (i.e., SNIP in the current study). Therefore, we have developed a novel method for the evaluation of FMI in piecemeal 
surgeries using a fluorescence grid analysis. Although in this study the TBRs of the fluorescence grid analysis were not substantially different from those of the standard analysis, the former method provided better insight in the variability of fluorescence signal. When evaluating FMI in piecemeal surgery, this variability of data is very important as the ultimate goal is to identify all tissue fragments that comprise the target tissue. When a suitable biomarker is identified through fluorescence grid analysis, the clinical value (i.e., in vivo contrast) can be assessed in a subsequent study.

Other studies that address the clinical problem of incomplete resection of SNIP have also reported the use of alternative imaging techniques to discriminate between SNIP and uninvolved tissue $[29,30]$. Yet, these imaging methods are mainly limited to morphological information and therefore lack specificity to distinguish SNIP from surrounding inflammatory tissue. Molecular imaging approaches may improve the specificity of contrast and thus harbor more clinical potential. Recently, the use of Raman spectroscopy showed $90 \%$ accuracy in distinguishing normal sinonasal mucosa, chronic rhinosinusitis, and SNIP tissue [31]. In contrast to Raman spectroscopy, a point measurement spectroscopy technique, FMI can visualize the complete mucosa of interest in vivo and can guide the surgeon during SNIP surgery rather than measure the surgeon's observations.

In the current study, considerable variability in fluorescent intensity was observed in both SNIP and uninvolved mucosa. Fluorescence signal was not only limited to SNIP but also occurred in regions that showed abundancy of plasma cells expressing VEGF-A and tissue showing reactive changes and edema. This moderate co-localization of bevacizumab-800CW and VEGF-A expression is in line with previous studies [21, 23, 32]. We surmise that this is because bevacizumab-800CW targets the extracellular matrix-bound isoform of VEGF-A, whereas immunohistochemical staining mainly detects an intracellular VEGF-A isoform [33, 34]. Secondly, and often underestimated in targeted imaging, nonspecific mechanisms, such as vascularity, vascular permeability, interstitial pressure, and internalization of the tracer, contribute to accumulation of the fluorescent tracer in the target tissue [35-37]. For instance, the increase in VEGF-A may cause increased vascular permeability in the inverted papilloma and adjacent inflammatory tissue $[38,39]$. Differentiating between nonspecific and specific accumulation of fluorescent tracers is one of the most important challenges in FMI today. Efforts that may solve this problem to better quantify receptor expression include paired-imaging agent strategies, which are now evaluated in preclinical studies [40, 41]. Although previous studies with bevacizumab-800CW showed tumor-specific fluorescence signal [10, 20, 22], we believe that for piecemeal SNIP surgery (in vivo) FMI using bevacizumab-800CW lacks sensitivity and specificity for SNIP surgery, which limits clinical applicability.

The performance of FMI in SNIP surgery can be improved in two ways. First, improved understanding of the poorly understood SNIP pathogenesis may help identify new biomarkers that are highly specific for SNIP tissue. Proteins involved in epithelial remodeling into SNIP may be of interest, of which the main etiologies include human papillomavirus [42, 43], chronic inflammation [44, 45], and angiogenic factors [19]. To further increase the possibility of detecting small SNIP residues, fluorescent tracers with an on/off mechanism could be exploited to achieve fluorescence signal in the target tissue only and increase contrast ${ }^{45}$. Technical challenges for in vivo endoscopic FMI remain due to a narrow field of view in the sinonasal anatomical area and the substantial bleeding that occurs during SNIP surgery, which obscures the optical signal and impedes in vivo contrast.

In conclusion, although SNIP tissue showed increased fluorescence signal compared to uninvolved mucosa, the clinical applicability of VEGF-targeted FMI is limited by low sensitivity and specificity to differentiate small tissue fragments. To overcome the problem of ex vivo tissue fragment analysis that occurs after piecemeal surgery, we have developed fluorescence grid analysis, a novel framework for the assessment of FMI. This method may serve as a guideline for future studies that evaluate FMI in piecemeal surgery using other disease targets. When more specific biomarkers for SNIP are discovered, a fluorescence grid analysis could more comprehensively inform about the clinical applicability of the fluorescent tracer studied in phase I studies, allowing for go/no-go decision in an early stage.

Supplementary Information The online version contains supplementary material available at https://doi.org/10.1007/s00259-021-05567-x.

Acknowledgements We thank all patients who participated in this study. We thank Maaike Barentsen for the assistance during tissue processing.

Author contribution JV, FJV, AGWKM, RAF, GMvD, BvdV, and MJHW designed the study. JV, JGdW, and FJV performed data acquisition, analyzed and interpreted data, and drafted the manuscript. WTH designed the fluorescence grid analysis. BvdV supervised the histopathological analyses. WBN was involved in the GMP production of bevacizumab-800CW. AGWKM and RAF performed surgical procedures. GMvD, BvdV, and MJHW supervised the study, interpreted data, and supervised writing of the manuscript. All the authors critically revised the manuscript.

Funding Open access funding was provided by the University of Groningen.

Data availability All data are available from the corresponding author (MJHW) on reasonable request. 


\section{Declarations}

Ethics approval The study protocol was approved by the institution's medical ethics committee, and the trial was performed in accordance with the principles of Good Clinical Practice guidelines and the Declaration of Helsinki.

Informed consent Signed written informed consent was obtained from all patients included in the study.

Conflict of interest GMvD is CEO, founder, and shareholder of TRACER Europe BV/AxelaRx. BvdV is member of the Scientific Advisory Board of Visiopharm for which compensation is received by the UMCG. The other authors declare no competing interests.

Open Access This article is licensed under a Creative Commons Attribution 4.0 International License, which permits use, sharing, adaptation, distribution and reproduction in any medium or format, as long as you give appropriate credit to the original author(s) and the source, provide a link to the Creative Commons licence, and indicate if changes were made. The images or other third party material in this article are included in the article's Creative Commons licence, unless indicated otherwise in a credit line to the material. If material is not included in the article's Creative Commons licence and your intended use is not permitted by statutory regulation or exceeds the permitted use, you will need to obtain permission directly from the copyright holder. To view a copy of this licence, visit http://creativecommons.org/licenses/by/4.0/.

\section{References}

1. Busquets JM, Hwang PH. Endoscopic resection of sinonasal inverted papilloma: a meta-analysis. Otolaryngol Head Neck Surg. 2006;134(3):476-82.

2. Lund VJ, Stammberger H, Nicolai P, et al. European position paper on endoscopic management of tumours of the nose, paranasal sinuses and skull base. Rhinol Suppl. 2010;22:1-143.

3. Wang MJ, Noel JE. Etiology of sinonasal inverted papilloma: a narrative review. World J Otorhinolaryngol Head Neck Surg. 2016;3(1):54-8.

4. Chiu AG, Jackman AH, Antunes MB, Feldman MD, Palmer JN. Radiographic and histologic analysis of the bone underlying inverted papillomas. Laryngoscope. 2006;116(9):1617-20.

5. Goudakos JK, Blioskas S, Nikolaou A, Vlachtsis K, Karkos P, Markou KD. Endoscopic resection of sinonasal inverted papilloma: systematic review and meta-analysis. Am J Rhinol Allergy. 2018;32(3):167-74.

6. Peng R, Thamboo A, Choby G, Ma Y, Zhou B, Hwang PH. Outcomes of sinonasal inverted papilloma resection by surgical approach: an updated systematic review and meta-analysis. Int Forum Allergy Rhinol. 2019;9(6):573-81.

7. Xiao-Ting W, Peng L, Xiu-Qing W, et al. Factors affecting recurrence of sinonasal inverted papilloma. Eur Arch Otorhinolaryngol. 2013;270(4):1349-53.

8. Vonk J, de Wit JG, Voskuil FJ, Witjes MJH. Improving oral cavity cancer diagnosis and treatment with fluorescence molecular imaging. Oral Dis. 2020.

9. Voskuil FJ, de Jongh SJ, Hooghiemstra WTR, et al. Fluorescenceguided imaging for resection margin evaluation in head and neck cancer patients using cetuximab-800CW: a quantitative doseescalation study. Theranostics. 2020;10(9):3994-4005.

10. Koller M, Qiu SQ, Linssen MD, et al. Implementation and benchmarking of a novel analytical framework to clinically evaluate tumor-specific fluorescent tracers. Nat Commun. 2018;9(1):3739-018-05727-y.

11. Gao RW, Teraphongphom NT, van den Berg NS, et al. Determination of tumor margins with surgical specimen mapping using near-infrared fluorescence. Cancer Res. 2018;78(17):5144-54.

12. Nagengast WB, Hartmans E, Garcia-Allende PB, et al. Nearinfrared fluorescence molecular endoscopy detects dysplastic oesophageal lesions using topical and systemic tracer of vascular endothelial growth factor A. Gut. 2019;68(1):7-10.

13. Jasper, Vonk Jaron G, de Wit Floris J, Voskuil Yang Hang, Tang Wouter TR, Hooghiemstra Matthijs D, Linssen Evert, van den Broek Jan J, Doff Sebastiaan AHJ, de Visscher Kees P, Schepman Bert, van der Vegt Gooitzen M, van Dam Max JH, Witjes. Epidermal growth factor receptor targeted fluorescence molecular imaging for postoperative lymph node assessment in patients with oral cancer. Journal of Nuclear Medicine. 2021. https://jnm.snmjournals.org/content/early/2021/09/15/jnumed. 121.262530 .

14. Yoon BN, Chon KM, Hong SL, et al. Inflammation and apoptosis in malignant transformation of sinonasal inverted papilloma: the role of the bridge molecules, cyclooxygenase-2, and nuclear factor $\kappa$ B. Am J Otolaryngol. 2013;34(1):22-30.

15. Katori H, Nozawa A, Tsukuda M. Markers of malignant transformation of sinonasal inverted papilloma. Eur J Surg Oncol. 2005;31(8):905-11.

16. Udager AM, Rolland DCM, McHugh JB, et al. High-frequency targetable EGFR mutations in sinonasal squamous cell carcinomas arising from inverted sinonasal papilloma. Cancer Res. 2015;75(13):2600-6.

17. Zhang J, Yang Y, Tang Y, Wu X, Cong L, Ruan B. The quantification and significance of muscle segment homeobox gene Msx2, human topoisomerase II- $\alpha$, HPV16 and VEGF in sinonasal inverted papilloma. Lin Chung Er Bi Yan Hou Tou Jing Wai Ke Za Zhi. 2014;28(23):1819-23.

18. Kong H, Guan Q, Sun X, Wang N. Significance and expression of VEGF and its receptor in nasal inverted papillomas and the correlation with malignant phenotype. Lin Chung Er Bi Yan Hou Tou Jing Wai Ke Za Zhi. 2012;26(8):337-8, 342.

19. Liu W, Li Z, Luo Q, et al. The elevated expression of osteopontin and vascular endothelial growth factor in sinonasal inverted papilloma and its relationship with clinical severity. Am J Rhinol Allergy. 2011;25(5):313-7.

20. Steinkamp PJ, Pranger BK, Li M, et al. Fluorescence-guided visualization of soft tissue sarcomas by targeting vascular endothelial growth factor-A: a phase 1 single-center clinical trial. J Nucl Med. 2020.

21. Lamberts LE, Koch M, de Jong JS, et al. Tumor-specific uptake of fluorescent bevacizumab-IRDye $800 \mathrm{CW}$ microdosing in patients with primary breast cancer: a phase I feasibility study. Clin Cancer Res. 2017;23(11):2730-41.

22. Hartmans E, Tjalma JJJ, Linssen MD, et al. Potential red-flag identification of colorectal adenomas with wide-field fluorescence molecular endoscopy. Theranostics. 2018;8(6):1458-67.

23. Tjalma JJJ, Koller M, Linssen MD, et al. Quantitative fluorescence endoscopy: an innovative endoscopy approach to evaluate neoadjuvant treatment response in locally advanced rectal cancer. Gut. 2020;69(3):406-10.

24. Ter Weele EJ, Terwisscha van Scheltinga AG, Linssen MD, et al. Development, preclinical safety, formulation, and stability of clinical grade bevacizumab-800CW, a new near infrared fluorescent imaging agent for first in human use. Eur J Pharm Biopharm. 2016; 104:226-234.

25. Linssen MD, Ter Weele EJ, Allersma DP, et al. Roadmap for the development and clinical translation of optical tracers cetuximab-800CW and trastuzumab-800CW. J Nucl Med. 2019;60(3):418-23. 
26. Koch M, Symvoulidis P, Ntziachristos V. Tackling standardization in fluorescence molecular imaging. Nat Photonics. 2018;12(9):505-15.

27. Kapoor S, Lu G, van den Berg, Nynke S, et al. Effect of formalin fixation for near-infrared fluorescence imaging with an antibody-dye conjugate in head and neck cancer patients. Molecular Imaging and Biology. 2020:1-7.

28. Óvári A, Starke N, Schuldt T, et al. Optical coherence tomography and confocal laser scanning microscopy as non-invasive tools in the diagnosis of sinonasal inverted papilloma: a pilot study. Eur Arch Otorhinolaryngol. 2018;275(7):1775-81.

29. Kidwai SM, Parasher AK, Schorn VJ, et al. Optical imaging with a high-resolution microendoscope to identify sinonasal pathology. Am J Otolaryngol. 2018;39(4):383-7.

30. Mascarella MA, Alrasheed A, Fnais N, et al. Raman spectroscopy for inverted papilloma: a proof-of-concept study. Otolaryngol Head Neck Surg. 2018;159(3):587-9.

31. Tjalma JJ, Garcia-Allende PB, Hartmans E, et al. Molecular fluorescence endoscopy targeting vascular endothelial growth factor A for improved colorectal polyp detection. J Nucl Med. 2016;57(3):480-5.

32. Nagengast WB, de Vries EG, Hospers GA, et al. In vivo VEGF imaging with radiolabeled bevacizumab in a human ovarian tumor xenograft. J Nucl Med. 2007;48(8):1313-9.

33. Stollman TH, Scheer MG, Leenders WP, et al. Specific imaging of VEGF-A expression with radiolabeled anti-VEGF monoclonal antibody. Int J Cancer. 2008;122(10):2310-4.

34. Tichauer KM, Wang Y, Pogue BW, Liu JT. Quantitative in vivo cell-surface receptor imaging in oncology: kinetic modeling and paired-agent principles from nuclear medicine and optical imaging. Phys Med Biol. 2015;60(14):R239-69.

35. Thurber GM, Dane WK. A mechanistic compartmental model for total antibody uptake in tumors. J Theor Biol. 2012;314:57-68.
36. Thurber GM, Weissleder R. Quantitating antibody uptake in vivo: conditional dependence on antigen expression levels. Mol Imaging Biol. 2011;13(4):623-32.

37. Apte RS, Chen DS, Ferrara N. VEGF in signaling and disease: beyond discovery and development. Cell. 2019;176(6):1248-64.

38. Ferrara N, Gerber HP, LeCouter J. The biology of VEGF and its receptors. Nat Med. 2003;9(6):669-76.

39. Tichauer KM, Samkoe KS, Gunn JR, et al. Microscopic lymph node tumor burden quantified by macroscopic dual-tracer molecular imaging. Nat Med. 2014;20(11):1348-53.

40. Samkoe KS, Park Y, Marra K, Chen EY, Tichauer KM. Pairedagent imaging for detection of head and neck cancers. Proc SPIE Int Soc Opt Eng. 2019;10853:https://doi.org/10.1117/12.25108 97.

41. Syrjänen K, Syrjänen S. Detection of human papillomavirus in sinonasal papillomas: systematic review and meta-analysis. Laryngoscope. 2013;123(1):181-92.

42. Ding R, Sun Q, Wang Y. Association between human papilloma virus infection and malignant sinonasal inverted papilloma. Laryngoscope. 2020.

43. Roh HJ, Procop GW, Batra PS, Citardi MJ, Lanza DC. Inflammation and the pathogenesis of inverted papilloma. Am J Rhinol. 2004;18(2):65-74.

44. Zhao L, Li CW, Jin P, et al. Histopathological features of sinonasal inverted papillomas in chinese patients. Laryngoscope. 2016;126(4):E141-7.

45. Voskuil FJ, Steinkamp PJ, Zhao T, et al. Exploiting metabolic acidosis in solid cancers using a tumor-agnostic $\mathrm{pH}$-activatable nanoprobe for fluorescence-guided surgery. Nat Commun. 2020;11(1):3257-020-16814-4.

Publisher's note Springer Nature remains neutral with regard to jurisdictional claims in published maps and institutional affiliations.

\section{Authors and Affiliations}

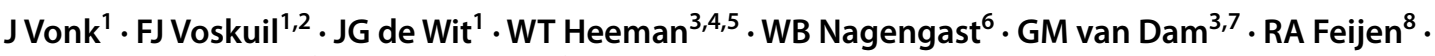 AGW Korsten-Meijer ${ }^{8} \cdot$ B van der Vegt $^{2} \cdot$ MJH Witjes $^{1}$}

1 Department of Oral \& Maxillofacial Surgery, University of Groningen, University Medical Center Groningen, PO Box 30.001, 9700 RB Groningen, the Netherlands

2 Department of Pathology \& Medical Biology, University of Groningen, University Medical Center Groningen, Groningen, the Netherlands

3 Medical Imaging Center, University of Groningen, University Medical Center Groningen, Groningen, the Netherlands

4 Faculty Campus Fryslân, University of Groningen, Leeuwarden, the Netherlands

5 LIMIS Development B.V, Leeuwarden, the Netherlands
6 Department of Gastroenterology and Hepatology, University of Groningen, University Medical Center Groningen, Groningen, the Netherlands

7 AxelaRx/TRACER B.V, Groningen, the Netherlands

8 Department of Otorhinolaryngology/Head and Neck Surgery, University Medical Center Groningen, University of Groningen, Groningen, the Netherlands 